\title{
Fetal Alcohol Exposure Impairs Alveolar Macrophage Function via Decreased Glutathione Availability
}

\author{
THERESA W. GAUTHIER, XIAO-DU PING, FRANK L. HARRIS, MICHAEL WONG, \\ HUSNI ELBAHESH, AND LOU ANN S. BROWN \\ Department of Pediatrics, Division of Neonatal-Perinatal Medicine, Emory University School of Medicine, \\ Atlanta, Georgia 30322
}

\begin{abstract}
Immature function of the alveolar macrophage increases the risk of pulmonary infections in premature newborns. In utero alcohol increases fetal systemic oxidative stress. Because the premature lung is deficient in glutathione (GSH), we hypothesized that chronic in utero alcohol (ethanol) exposure exacerbates the oxidative stress within the developing lung, thereby impairing alveolar macrophage function. Additionally, we evaluated the effects of in vivo and in vitro GSH availability on ethanol-exposed macrophage function. Using a guinea pig model of chronic in utero ethanol exposure, fetal epithelial lining fluid (ELF) and alveolar macrophage GSH were decreased with increased markers of oxidative stress. Ethanol-exposed macrophage exhibited impaired phagocytosis and increased apoptosis compared with gestational control. When the GSH precursor S-adenosyl-methionine (SAM) was added to the maternal drinking water containing ethanol, fetal ELF and macrophage GSH were maintained and ELF oxidative stress diminished. In vivo maternal SAM therapy maintained macrophage phagocytosis and decreased apoptosis. In vitro GSH supplements also improved
\end{abstract}

\section{ABSTRACT}

phagocytosis and viability in both premature and ethanolexposed macrophage. This suggested that in utero ethanol impaired premature macrophage function and viability via decreased GSH availability. Furthermore, GSH supplementation during and after ethanol exposure improved fetal macrophage function and viability. These results add a new dimension to the detrimental effects of fetal alcohol exposure on the developing alveolar macrophage, raising the possibility of GSH therapy to augment premature alveolar macrophage function. (Pediatr Res 57: 76-81, 2005)

ELF, epithelial lining fluid

\section{Abbreviations}

GSH, glutathione

GSSG, oxidized glutathione

HNE, 4-hydroxynonenal

MDA, malonyldialdehyde

PARP, poly (ADP-ribose) polymerase

SAM, S-adenosyl-methionine
Fetal alcohol exposure remains a significant problem in our society. Alcohol abuse and binge drinking by pregnant women has been estimated at an alarming $35 \%$ of pregnancies (1-3). Fetal alcohol syndrome or alcohol-related neurodevelopmental disorder has been estimated to occur in $\sim 1 / 1000$ pregnancies (4). Because there is a strong relationship between cocaine abuse and concurrent alcohol ingestion (5-7), and both substances may increase the risk of premature delivery $(7,8)$, a significant population of premature infants is exposed to alcohol in utero.

In animal models of fetal alcohol exposure, alcohol increases systemic oxidative stress in the developing fetus (914). A decrease in the antioxidant GSH has been demonstrated,

Received November 24, 2003; Accepted July 12, 2004.

Correspondence: Theresa W. Gauthier, M.D., Department of Pediatrics, Emory University, 2040 Ridgewood Dr. NE, Atlanta, GA 30322; e-mail: tgauthi@emory.edu

Supported by National Institutes of Health grants HD39651 and R01 AA013979, the American Lung Association of Georgia, and the Emory Medical Care Foundation Faculty Scholar Award (T.W.G.).

DOI: 10.1203/01.PDR.0000149108.44152.D3 particularly in the alcohol-exposed fetal liver $(9,15,16)$. As an essential antioxidant in the body, GSH is normally present in high concentrations in the ELF of the lung (17). The fetal lung is at risk for increased oxidative stress during development, because maturation of antioxidant systems, including GSH, is gestationally dependent $(18,19)$. A reduction in alveolar GSH, as seen in the premature infant, leaves the preterm lung susceptible to increased pulmonary oxidative injury $(20,21)$ and chronic lung disease $(18,22)$. The impact of the oxidative stress of fetal alcohol exposure superimposed on the low GSH environment of the developing lung has received little attention.

The immature alveolar macrophage's functions contribute to an increased risk of infections in the premature lung. When compared with the adult, the newborn macrophage functions of chemotaxis, phagocytosis, and bacterial killing are diminished (23-25). GSH is essential for optimal functioning of phagocytic cells, such as the alveolar macrophage. For neutrophils, phagocytosis is impaired in many clinical states associated with decreased GSH (26), and the essential role for GSH is supported by the ability of in vitro GSH to normalize intracel- 
lular GSH pools and phagocytosis (27). Thus, we hypothesized that the exaggerated oxidative stress associated with in utero alcohol (ethanol) exposure would decrease GSH availability for the alveolar macrophage in the developing lung. Furthermore, we postulated that diminished GSH availability and the corresponding oxidant stress would further impair the function of ethanol-exposed premature alveolar macrophage.

By using a guinea pig model of fetal alcohol exposure, the purpose of this study was to examine the effects of chronic in utero ethanol exposure on GSH availability in the premature lung and the resulting impact on fetal alveolar macrophage function. The second goal was to determine whether GSH supplements, in vitro or in vivo, would maintain or restore fetal alveolar macrophage function.

\section{MATERIALS AND METHODS}

Guinea pig model of fetal ethanol exposure. Timed-pregnant pathogenfree guinea pigs (Harlan Bioproducts for Science, Indianapolis, IN) were shipped on $\sim \mathrm{d} 35$ gestation (term, $\sim 71 \mathrm{~d}$ ) and randomly assigned to ethanol or no ethanol in the drinking water with incremental increases up to $4 \%$ ethanol $(28-30)(25 \%$ calories $+8 \mathrm{mg} / 100 \mathrm{~mL}$ saccharin) by $\sim \mathrm{d} 40$ gestation. The ethanol was increased in increments to avoid decreased food intake or loss of pregnancy secondary to inadequate caloric intake. The only access to drinking water was the experimental mixture. Solid diet was provided ad libitum to the ethanol group, whereas the control dams were pair-fed solid diet to match the ethanol dam. In initial studies using this guinea pig model, we found that the solid food intake was significantly less in the ethanol-fed dams $(28-30 \mathrm{~g} / \mathrm{d})$ compared with the ad libitum control dams (45-50 g/d). Therefore, we initiated this pair-fed model of fetal ethanol exposure in attempts to eliminate nutrition as a confounding factor in the experimental design and present data only from pair-fed controls. Where appropriate, the GSH precursor SAM (Ptoluenesulfonate salt, $1.0 \mathrm{mg} / \mathrm{mL}$; Sigma Chemical Co., St. Louis, MO) was added to the drinking water containing the ethanol. The experimental drinking water was replenished every other day and the SAM solution prepared twice a week. The fetuses were then removed by cesarean section on d 55 of gestation, corresponding to $\sim 30$ wk human gestation. Serum alcohol levels drawn at approximately $0900 \mathrm{~h}$ were $\sim 0.05 \pm 0.01 \%$ in both the mother and the pup. This level approaches legal intoxication in the state of Georgia $(0.07 \%)$. All animals were used in accordance with the National Institutes of Health Guidelines (Guide for the Care and Use of Laboratory Animals), with protocols reviewed and approved by the Emory University Institutional Animal Care Committee.

Alveolar macrophage isolation. After delivery, the pups were anesthetized with sodium pentobarbital intraperitoneally and the trachea was cannulated for lavage and collection of alveolar macrophage. The fetal lungs were lavaged with $1.5 \mathrm{~mL}$ sterile $\mathrm{PBS}\left(37^{\circ} \mathrm{C}, \mathrm{pH} 7.4\right)$. The sample was centrifuged at 1200 rpm for 8 min and the cell pellet resuspended in Dulbecco's modified Eagle's medium (DMEM)-F-12 media with $2 \%$ fetal bovine serum, with penicillin and streptomycin (100 u/L each). The supernatant of the ELF was saved for further analysis (see below). Cells in the lavage fluid were predominantly macrophage $(>95 \%)$, with viability of $>95 \%$ as determined by calcein/ethidium iodide "live-dead" stain. This method routinely obtained $10^{6}$ pooled macrophage/ litter, with each litter representing $n=1$.

Glutathione analysis. ELF and macrophage GSH and GSSG were determined by HPLC analysis as previously described by this laboratory (31-33). Briefly, after isolation the sample was immediately acidified with perchloric acid (5\% total) and $50 \mathrm{mM}$ (final) $\gamma$-glutamyl-glutamate (an internal standard) added. Fractions were derivatized with iodoacetic acid and dansyl chloride and then the GSH and GSSG fractions separated by HPLC on an amino $\mu$ BondaPak column (Waters, Milford, MA). To control for dilution by the lavage procedure, the GSH and GSSG content of the ELF was corrected by the urea method (31-33). For alveolar macrophage measurements, GSH and GSSG values were normalized to cell number. Fluorescent detection was used to separate and quantitate the dansyl derivatives relative to the fluorescence of the $\gamma$-glutamyl-glutamate standard.
ELF oxidative stress. To further evaluate the ELF for signs of oxidative stress, we measured the lipid peroxidation product HNE via commercially available ELISA (Research Diagnostics, Flanders, NJ). Values for HNE were normalized to ELF IgA, a secretory product not altered by injury (34-36). The ELF IgA was measured by ELISA with the primary antibody obtained from Sigma Chemical Co.

Phagocytosis. After isolation, macrophage were plated at $10^{6} \mathrm{cell} / \mathrm{mL}$ in DMEM-F-12 media with $2 \%$ fetal bovine serum, penicillin plus streptomycin and cultured at $37^{\circ} \mathrm{C}, 5 \% \mathrm{CO}_{2}$. Supplemental GSH $(200 \mu \mathrm{M})$ was added when appropriate to the culture media. After $2 \mathrm{~h}$, alveolar macrophages were washed, FITC-labeled inactivated Staphylococcus aureus (Molecular Probes, Eugene, OR) was added in a 1:1 ratio (alveolar macrophage:bacteria), and the cells were cultured for an additional $2 \mathrm{~h}$ (37). After the incubation period, cells were washed and phagocytosis of $S$. aureus was determined via quantitative digital analysis of fluorescence (QImaging, Burnaby, BC, Canada) with data analysis via Image-Pro Plus for Windows. Values are presented as mean relative fluorescent units $(\mathrm{RFU}) / \mathrm{cell} \pm \mathrm{SEM}$ and the mean percentage of cells fluorescently positive \pm SEM as tallied from at least 10 experimental fields per set.

Apoptosis. After culture for $4 \mathrm{~h}$, macrophages were fixed with $3.7 \%$ paraformaldehyde and nonspecific binding blocked with BSA. Apoptosis was determined by staining for the cleaved PARP, and for DNA fragmentation via terminal dUTP nick-end labeling (TUNEL). The primary antibody for cleaved PARP (Oncogene, Cambridge, MA) was added in a 1:100 dilution and the sample incubated for $2 \mathrm{~h}$. Cells were serially rinsed with PBS and the secondary antibody (anti-rabbit IgG, Alexa Fluor, Molecular Probes, Eugene, OR) added in a 1:200 dilution for $1 \mathrm{~h}$. For the TUNEL analysis, fixed cells were permeabilized with $0.1 \%$ Triton $\times 100$ and $0.1 \%$ sodium citrate and then incubated for $1 \mathrm{~h}$ with TMR-Red (in situ cell death detection kit, Roche Molecular Biochemicals, Indianapolis, IN). Cellular fluorescence was determined via quantitative digital analysis via Image-Pro Plus for Windows. Values are presented as the mean percentage of cells fluorescently positive \pm SEM as tallied from at least 10 experimental fields per set.

Macrophage oxidative stress. After culture for $4 \mathrm{~h}$, macrophages were fixed with $3.7 \%$ paraformaldehyde and nonspecific binding blocked with BSA. Oxidative stress was evaluated by determining the presence of the fatty acid oxidation products HNE and MDA (12) via immunohistochemistry. The primary antibodies were added in a 1:100 dilution (MDA, Academy Biomedical, Houston, TX; HNE, Alexis, San Diego, CA) and the sample incubated for $2 \mathrm{~h}$. Cells were serially rinsed with PBS and the secondary fluorescent antibody (or peroxidase antibody) (Sigma Chemical Co.) added in a 1:200 dilution for $1 \mathrm{~h}$. Macrophage staining was determined via quantitative digital analysis via Image-Pro Plus for Windows. Values are presented as mean RFU/cell \pm SEM for MDA and mean relative units (RU)/cell \pm SEM for HNE as tallied from at least 10 experimental fields per set.

Statistical analysis. SigmaStat for Windows (Systat Software, Inc., Point Richmond, CA) was used for statistical calculations. ANOVA was used to detect overall differences. Statistical differences between groups were detected by posthoc analysis (Student-Newman-Keuls) and a $p \leq 0.05$ was considered significant.

\section{RESULTS}

In utero ethanol exposure decreased ELF GSH availability. Fetal ELF GSH was significantly decreased after in utero ethanol exposure ( $p<0.05$, Fig. $1 A)$, with a corresponding increase in the oxidized portion of GSSG when compared with gestational controls $(p<0.05$, Fig. 1B). Therefore, in utero ethanol increased the oxidative stress of the fetal airway as indicated by decreased GSH and increased GSSG/(GSSG + GSH). The addition of the GSH precursor SAM to the maternal diet during ethanol ingestion maintained fetal ELF GSH and decreased oxidized GSSG $(p<0.05$ versus ethanol, Fig. 1, A and $B$, respectively).

In utero ethanol increased products of oxidative stress in the ELF. The lipid peroxidation product HNE was significantly increased in the fetal ELF after in utero ethanol expo- 

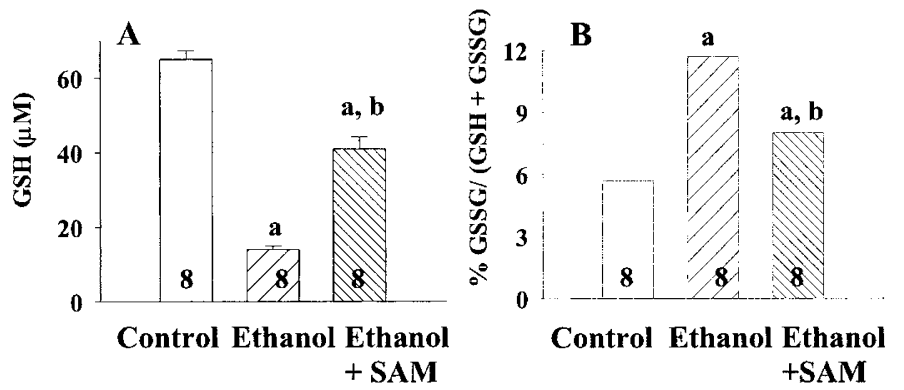

Figure 1. ELF GSH $(A)$ and percentage GSSG $(B)$. Timed-pregnant guinea pigs were randomly assigned to ethanol or no ethanol in the drinking water (4\%) and the groups pair-fed to match the ethanol dam. Where appropriate, SAM $(1.0 \mathrm{mg} / \mathrm{mL})$ was added to the drinking water. Pups were delivered by cesarean section on d 55 and ELF was collected by bronchoalveolar lavage. ELF GSH and GSSG were determined via HPLC analysis. Bar heights represent mean values \pm SEM of eight separate litters. ${ }^{a} p<0.05$ compared with control, ${ }^{\mathrm{b}} p<0.05$ compared with ethanol.

sure ( $p<0.05$ versus control, Fig. 2). When dams were given ethanol + SAM, the expected increase in free HNE in the fetal ELF due to in utero ethanol exposure was significantly attenuated ( $p<0.05$ versus ethanol, Fig. 2).

Decreased ELF GSH corresponded with altered alveolar macrophage GSH. Because in utero ethanol exposure decreased GSH availability and increased oxidative stress in the fetal ELF, we investigated whether this was accompanied by decreased GSH within the fetal alveolar macrophage. Fetal alveolar macrophage were isolated and GSH and GSSG measured via HPLC (33). In utero ethanol exposure decreased the fetal macrophage GSH/GSSG ratio by $\sim 73 \%$ ( $p<0.05$ versus control, Fig. 3). The addition of SAM to the maternal diet during ethanol ingestion maintained macrophage GSH/GSSG at control levels $(p<0.05$ versus ethanol, Fig. 3$)$.

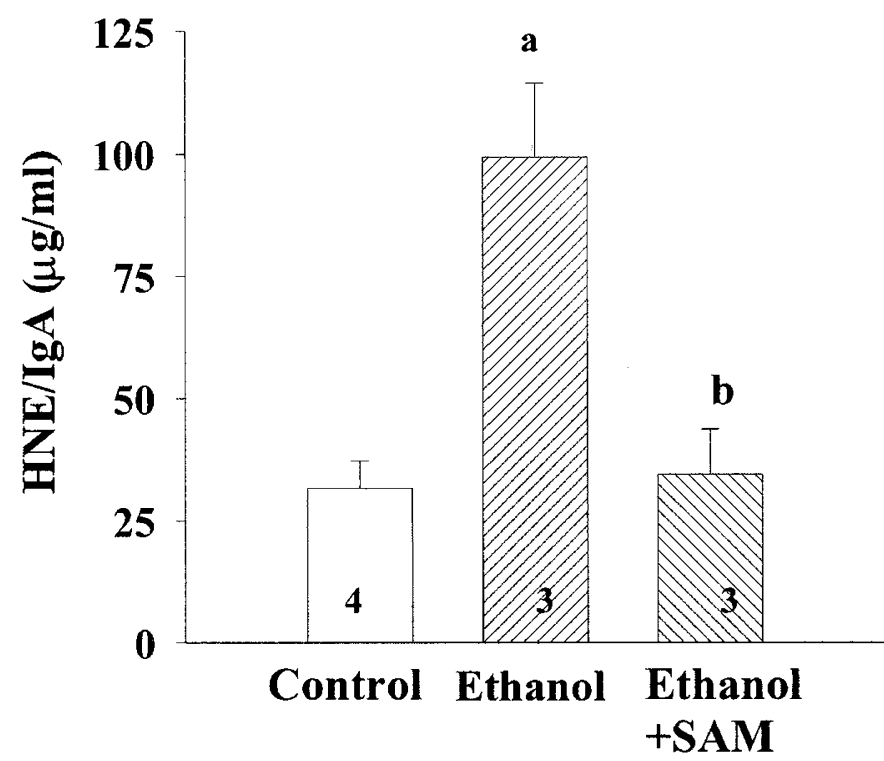

Figure 2. Lipid peroxidation product HNE in the ELF. Pups were delivered by cesarean section on d 55 after exposure to the experimental diet, and ELF was collected by bronchoalveolar lavage. Free HNE was measured via ELISA with normalization to ELF IgA. Bar heights represent mean values $(\mu \mathrm{g} / \mathrm{mL}) \pm$ SEM of $n$ separate litters. ${ }^{\mathrm{a}} p<0.05$ compared with control, ${ }^{\mathrm{b} p}<0.05$ compared with ethanol.

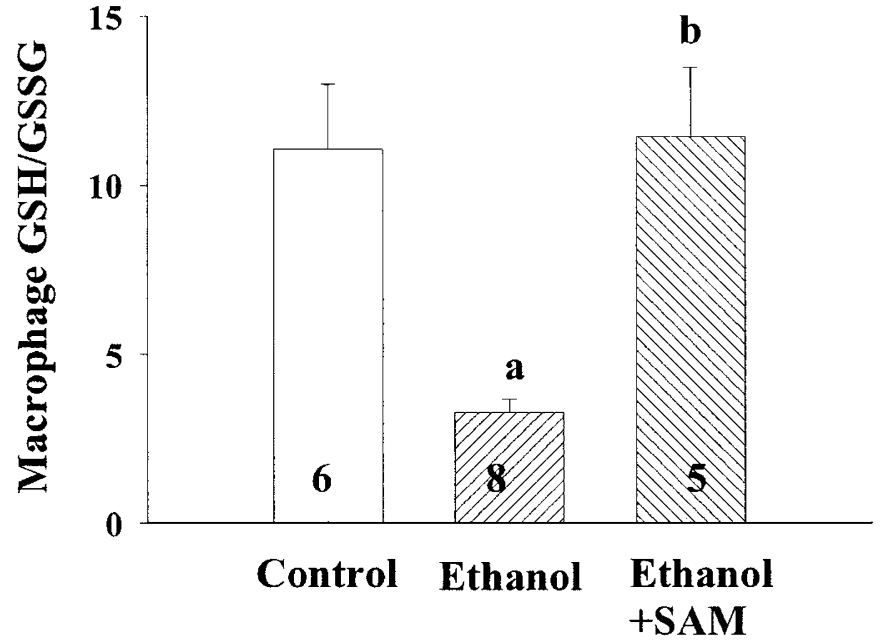

Figure 3. Fetal macrophage GSH/GSSG ratios. After cesarean section on d 55 gestation, fetal macrophage were isolated by bronchoalveolar lavage. Macrophage GSH and GSSG were determined via HPLC. Bar heights represent mean values \pm SEM of $n$ litters. ${ }^{a} p<0.05$ compared with control, ${ }^{\mathrm{b}} p<$ 0.05 compared with ethanol.

In utero ethanol increased lipid peroxidation in the alveolar macrophage. To further verify that in utero ethanol exposure increased oxidative stress in the fetal alveolar macrophage, the cells were evaluated for the presence of HNE and MDA, lipid peroxidation by-products. In utero ethanol exposure increased MDA staining on the macrophage by $>150 \%$ ( $p$ $<0.05$ versus control, Fig. 4A), whereas HNE staining was increased by $>80 \%$ ( $p<0.05$ versus control, Fig. $4 B$ ). Concurrent treatment with the glutathione precursor SAM during the ethanol ingestion normalized both MDA staining per cell and HNE staining per cell to control levels $(p<0.05$ versus ethanol, Fig. 4, $A$ and $B$, respectively).

Fetal ethanol exposure impaired premature macrophage phagocytosis. When compared with the macrophages from pair-fed gestational controls, the mean phagocytosis per cell was decreased by $\sim 30 \%$ after in utero ethanol exposure ( $p<$ 0.05 versus control, Fig. $5 A$ ). Ethanol exposure also reduced the percentage of cells positive for ingested $S$. aureus by

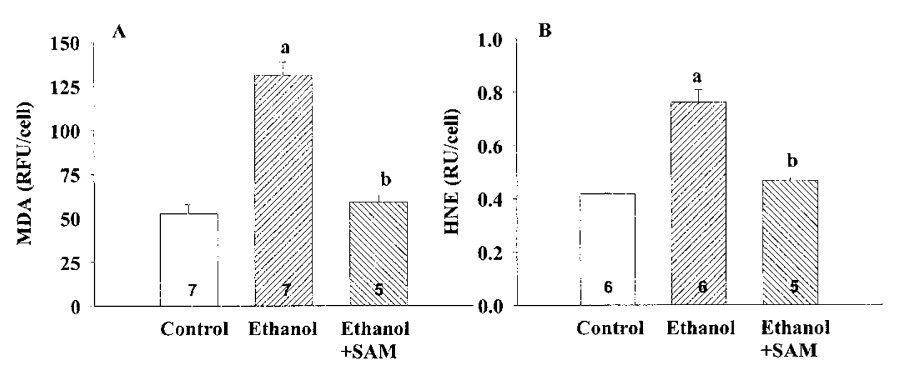

Figure 4. Lipid peroxidation products in the fetal macrophage. Fetal macrophage were isolated by bronchoalveolar lavage and cultured for $4 \mathrm{~h}$. Cells were fixed with $3.7 \%$ paraformaldehyde and then incubated with the primary antibody for MDA $(A)$ and HNE $(B)$. Cells were then stained with a secondary fluorescent antibody or a secondary peroxidase antibody. For MDA staining (A), bar heights represent mean relative fluorescent units (RFU/cell) \pm SEM of $n$ litters. For HNE staining $(B)$, bar heights represent mean relative staining (RU/cell) \pm SEM of $n$ litters. ${ }^{\mathrm{a}} p<0.05$ compared with control, ${ }^{\mathrm{b}} p<0.05$ compared with ethanol. 


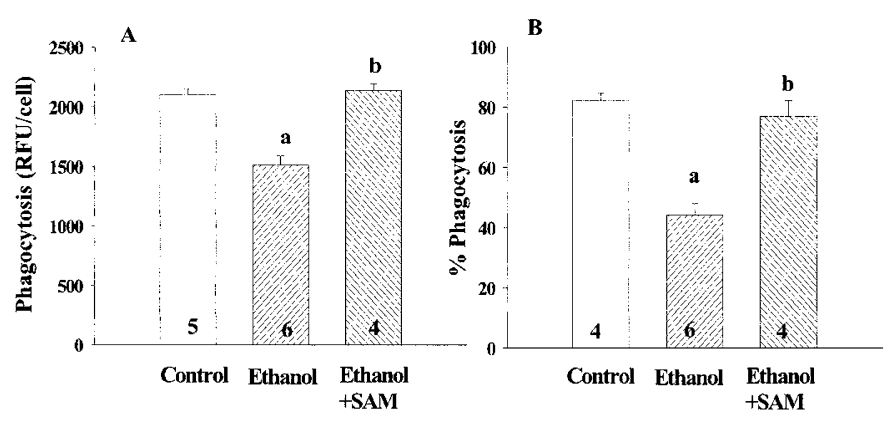

Figure 5. Baseline fetal macrophage phagocytosis. Fetal macrophages were isolated on d 55 by bronchoalveolar lavage and cultured for $2 \mathrm{~h}$. Cells were incubated with FITC-labeled inactivated $S$. aureus (1:1 ratio) for an additional $2 \mathrm{~h}$. Fluorescence was determined by quantitative digital analysis (Q imaging). Bar heights represent $(A)$ mean relative fluorescent units (RFU/cell) \pm SEM and $(B)$ percentage phagocytosis \pm SEM of $n$ litters. ${ }^{a} p<0.05$ compared with control, ${ }^{\mathrm{b}} p<0.05$ compared with ethanol.

$\sim 50 \%$ ( $p<0.05$ versus control, Fig. $5 B$ ). The GSH precursor SAM maintained both the mean phagocytosis per cell at control values ( $p<0.05$ versus ethanol, $p=$ NS versus control, Fig. 5A) as well as the percentage of macrophage positive for phagocytosis $(p<0.05$ versus ethanol, $p=$ NS versus control, Fig. 5B).

In utero ethanol exposure decreased fetal alveolar macrophage survival. Because of the decreased GSH availability for the ethanol-exposed macrophage and the dramatic reduction in cell phagocytosis, we postulated that the dysfunction of the ethanol-exposed macrophage was due to increased apoptotic cell death. To determine apoptosis, cells were stained after $4 \mathrm{~h}$ culture for the cleavage of PARP by caspase-3, an early indicator of the apoptosis pathway (38), and for DNA fragmentation via the TUNEL assay. Fetal ethanol exposure significantly increased the percentage of cells staining positive for PARP by more than 2-fold ( $p<0.05$ versus control, Fig. 6A). Similarly, TUNEL-positive cells were significantly increased in the ethanol group compared with control ( $p<0.05$ versus control, Fig. $6 B$ ). The addition of SAM normalized the percentage of cells with any staining for PARP to control values and significantly diminished TUNEL staining ( $p<0.05$ versus ethanol, respectively, Fig. 6, $A$ and $B$ ). These results suggested

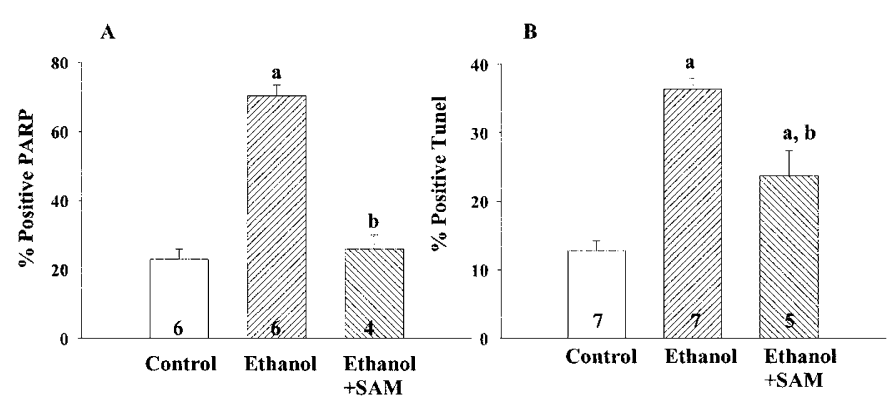

Figure 6. Baseline apoptosis in fetal macrophage. Fetal macrophage were isolated by bronchoalveolar lavage and cultured for $4 \mathrm{~h}$. Cells were fixed with $3.7 \%$ paraformaldehyde and then evaluated for apoptosis by staining for cleaved PARP $(A)$ and DNA fragmentation (TUNEL, $B$ ). Bar heights represent the percentage of positive cells \pm SEM of $n$ litters. ${ }^{a} p<0.05$ compared with control, ${ }^{\mathrm{b}} p<0.05$ compared with ethanol. that GSH availability in the ELF protected the fetal macrophage from ethanol-induced apoptosis.

In vitro GSH attenuated macrophage oxidative stress and improved function and viability. Given the role of extracellular GSH availability in macrophage oxidative stress, function, and viability, the possibility of macrophage rescue by in vitro GSH supplements was addressed. After isolation, supplemental GSH $(200 \mu \mathrm{M})$ was added to the culture media and macrophage oxidative stress, as determined by HNE and MDA staining, phagocytosis, and apoptosis were evaluated. In vitro GSH supplementation significantly diminished MDA per cell in the ethanol-exposed macrophage $(p<0.05$ compared with ethanol without GSH, Fig. 7A). Similarly, HNE staining was significantly attenuated by in vitro GSH supplementation in the ethanol macrophage $(p<0.05$ compared with ethanol without GSH, Fig. 7B).

Furthermore, the addition of GSH in vitro improved the mean phagocytosis per cell (Fig. 8A) and the percentage phagocytosis (Fig. $8 B$ ) in both the premature control macrophages and the ethanol-exposed macrophages ( $p<0.05$ compared with control without GSH, $p<0.05$ compared with ethanol without GSH, Fig. 8, $A$ and $B$ ).

Finally, in vitro GSH significantly reduced PARP-positive cells in control premature cells $(p<0.05$ compared with control without GSH, Fig. 9A). For the macrophages exposed to ethanol in utero, in vitro GSH significantly reduced the percentage of apoptotic cells as determined by both PARP staining ( $p<0.05$ compared with ethanol without GSH, Fig. $9 A)$ and TUNEL staining $(p<0.05$ compared with ethanol without GSH, Fig. 9B).

\section{DISCUSSION}

A growing body of clinical and experimental evidence has demonstrated that the chronic oxidative stress of alcohol exposure decreases the availability of the antioxidant GSH in the adult lung (39) and independently increases the risk and severity of acute respiratory distress syndrome (40). Indeed, experimental studies from our laboratories investigating the adult

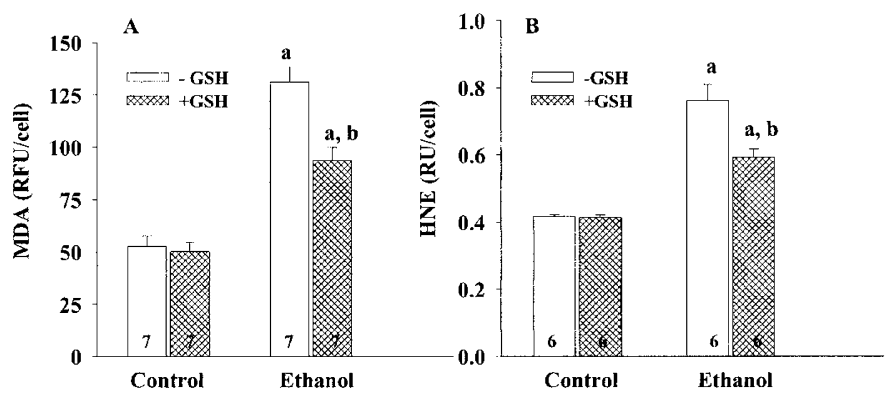

Figure 7. Macrophage oxidative stress with in vitro GSH supplements. Fetal macrophage were isolated by bronchoalveolar lavage and cultured with \pm 200 $\mu \mathrm{M}$ GSH for $2 \mathrm{~h}$. Cells were fixed with $3.7 \%$ paraformaldehyde and then incubated with the primary antibody for MDA $(A)$ and $\operatorname{HNE}(B)$. Cells were then stained with a secondary fluorescent antibody or a secondary peroxidase antibody. For MDA staining $(A)$, bar heights represent mean relative fluorescent units (RFU/cell) \pm SEM of $n$ litters. For HNE staining $(B)$, bar heights represent mean relative staining (RU/cell) \pm SEM of $n$ litters. ${ }^{a} p<0.05$ compared with control without GSH, ${ }^{\mathrm{b}} p<0.05$ compared with ethanol without GSH. 


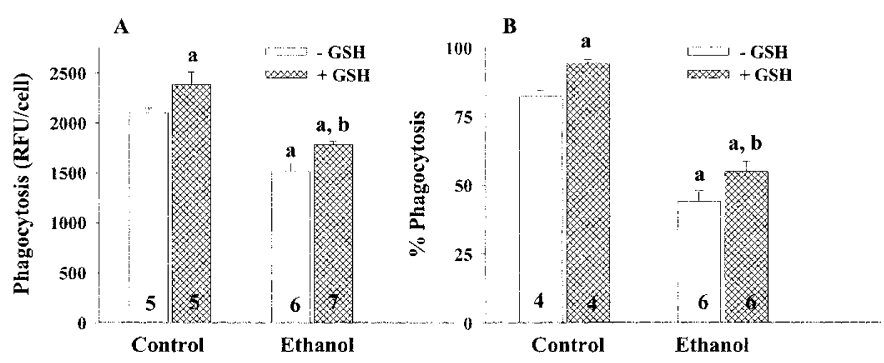

Figure 8. Macrophage phagocytosis with in vitro GSH supplementation. Fetal macrophage were isolated by bronchoalveolar lavage and cultured with $\pm 200 \mu \mathrm{M}$ GSH for $2 \mathrm{~h}$. FITC-labeled inactivated S. aureus was added to the media for an additional $2 \mathrm{~h}$. Fluorescence was determined by quantitative digital analysis. Bar heights represent $(A)$ mean relative fluorescent units $(\mathrm{RFU} /$ cell $) \pm \mathrm{SEM}$ and $(B)$ percentage phagocytosis \pm SEM of $n$ litters. ${ }^{\mathrm{a}} p<$ 0.05 compared with control without GSH, ${ }^{\mathrm{b}} p<0.05$ compared with ethanol without GSH.

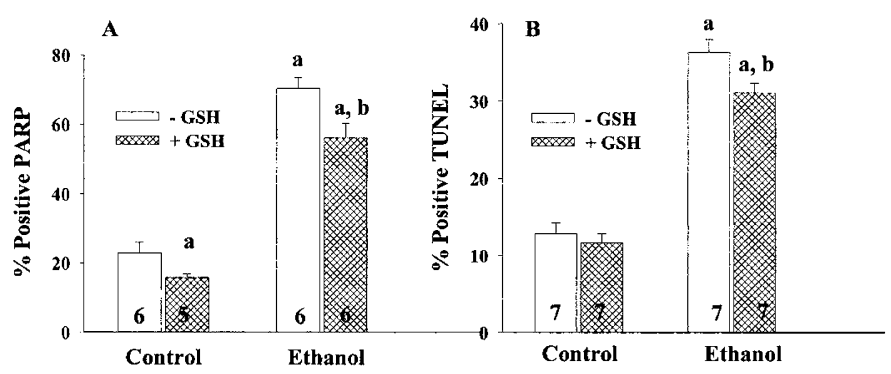

Figure 9. Apoptosis of the fetal macrophage after in vitro GSH supplementation. Fetal macrophage were cultured with $\pm 200 \mu \mathrm{M}$ GSH for $4 \mathrm{~h}$. To determine apoptosis, cells were fixed with $3.7 \%$ paraformaldehyde and then evaluated for cleaved PARP and DNA fragmentation (TUNEL). Fluorescence was determined via quantitative digital analysis. Bar heights represent $(A)$ percentage positive PARP \pm SEM and $(B)$ percentage positive TUNEL \pm SEM of $n$ litters. ${ }^{\mathrm{a}} p<0.05$ compared with control without GSH, ${ }^{\mathrm{b}} p<0.05$ compared with ethanol without GSH.

"alcoholic lung" have demonstrated that GSH homeostasis is impaired, with decreased GSH and increased oxidized GSSG in the ELF $(41,42)$. This state of chronic oxidative stress results in increased acute lung injury with sepsis, impaired type II epithelial cell function (42), and increased sensitivity to oxidant-induced apoptosis (33).

Prematurity is associated with decreased GSH availability in the ELF and decreased alveolar macrophage phagocytosis $(22,23)$. The current study shows that, similar to the adult, the fetus exposed to ethanol in utero demonstrated decreased GSH availability in the ELF compared with the pair-fed control. This was accompanied by increased oxidant stress in the ELF as assessed by diminished GSH/GSSG and increased lipid peroxidation. This ethanol-induced chronic oxidative stress in the ELF was coupled with oxidative stress in the alveolar macrophages as assessed by decreased GSH/GSSG and increased lipid peroxidation (MDA and HNE). Such chronic oxidative stress had physiologic ramifications for the alveolar macrophage, including decreased function such as phagocytosis and diminished cellular viability, when compared with cells from gestationally matched pair-fed control pups.

The increased macrophage apoptosis demonstrated after chronic in utero ethanol parallels the oxidant-induced injury and apoptosis described in other organs models of fetal alcohol syndrome, such as the developing brain (13) and liver (43). Because the entire fetus is exposed to the oxidative stress of in utero ethanol exposure, our current results suggest that the developing resident alveolar macrophage within the lung is similarly vulnerable to the toxicity of in utero ethanol.

Antioxidant replacement has been described as beneficial to other cell systems exposed to the chronic oxidative stress of fetal alcohol exposure. Replacement of GSH or vitamin E in the developing ethanol-exposed fetus has restored fetal growth (44), blunted neurologic injury (45), and preserved hepatic cell function $(46,47)$. In the current study, maintaining GSH availability in vivo via the maternal GSH donor SAM during ethanol ingestion protected the fetal lung and alveolar macrophage from oxidative stress. This was evident by attenuation of lipid peroxidation, as well as increased GSH/GSSG in the both the ELF and the alveolar macrophages. Maintenance of GSH status corresponded with protection of the developing macrophage from ethanol-induced dysfunction, with increased phagocytosis and decreased apoptosis. These results suggested that ethanol-induced decreases in GSH availability to the fetal lung were a central feature in the ethanol toxicity to the alveolar macrophages. Furthermore, increasing GSH availability in vitro improved control pair-fed fetal macrophage phagocytosis and blunted ethanol-induced dysfunction, in part by reducing apoptosis in the cell.

We chose to examine macrophage function at one point in gestation after chronic ethanol exposure. At this point ( $\sim 30 \mathrm{wk}$ of human gestation), GSH remains deficient in the developing fetus, as levels normally increase with gestation, with a surge at the time of term delivery (18). In our guinea pig model, the pair-fed control premature macrophage demonstrated cellular vulnerability, as evidenced by the presence of oxidative stress and apoptosis. Pair-feeding and diminished maternal food intake in the control dams may have contributed to the cellular vulnerability seen in the control premature macrophage. The additional oxidative stress of ethanol exposure augmented this vulnerability. Premature newborns are often exposed to increased oxidative stress in the lung due to the need for oxygen and/or ventilator therapy. Additional studies are necessary to evaluate whether the detrimental effects of ethanol-induced oxidative stress on the developing alveolar macrophage persist throughout gestation, especially at term when the GSH system approaches maturation.

In summary, this study demonstrates a novel deleterious effect of fetal ethanol exposure on the function and viability of the developing alveolar macrophage. In utero ethanol impaired macrophage function in part via decreased GSH availability in the lung. Maternal GSH supplementation during ethanol exposure and in vitro GSH supplementation after ethanol exposure protected the developing macrophage from apoptosis and dysfunction. The clinical implications and significance of these findings for the premature newborn's risk of infection and infectious complications remain under investigation. We hypothesize that the need for GSH availability in alveolar macrophage functioning can be applicable to other conditions in pregnancy that increase oxidative stress for the developing fetus. These include diabetes $(48,49)$, maternal smoking (50), pregnancy-induced hypertension (50-52), intrauterine growth retardation (53), and preterm premature rupture of membranes (54). Therefore, the potential implica- 
tions of these results have a broader therapeutic potential than maternal alcohol abuse alone. Moreover, this study provides important evidence for the need to identify the alcohol-exposed premature newborn. Future studies are necessary to explore a potential role of GSH availability to augment macrophage function in premature newborns under exaggerated oxidative stress, such as those exposed to alcohol in utero.

\section{REFERENCES}

1. Ebrahim SH, Diekman ST, Floyd RL, Decoufle P 1999 Comparison of binge drinking among pregnant and nonpregnant women, United States, 1991-1995. Am J Obstet Gynecol 180:1-7

2. Ebrahim SH, Luman ET, Floyd RL, Murphy CC, Bennett EM, Boyle CA 1998 Alcohol consumption by pregnant women in the United States during 1988-1995. Obstet Gynecol 92:187-192

3. Lester BM, ElSohly M, Wright LL, Smeriglio VL, Verter J, Bauer CR, Shankaran S, Bada HS, Walls HH, Huestis MA, Finnegan LP, Maza PL 2001 The maternal lifestyle study: drug use by meconium toxicology and maternal self-report. Pediatrics 107:309-317

4. Sampson PD, Streissguth AP, Bookstein FL, Little RE, Clarren SK, Dehaene P, Hanson JW, Graham JM Jr 1997 Incidence of fetal alcohol syndrome and prevalence of alcohol-related neurodevelopmental disorder. Teratology 56:317-326

5. Burkett G, Yasin S, Palow D 1990 Perinatal implications of cocaine exposure. J Reprod Med 35:35-42

6. Singer LT, Yamashita TS, Hawkins S, Cairns D, Baley J, Kliegman R 1994 Increased incidence of intraventricular hemorrhage and developmental delay in cocaineexposed, very low birth weight infants. J Pediatr 124:765-771

7. Spence MR, Williams R, DiGregorio GJ, Kirby-McDonnell A, Polansky M 1991 The relationship between recent cocaine use and pregnancy outcome. Obstet Gynecol 78:326-329

8. Lundsberg LS, Bracken MB, Saftlas AF 1997 Low-to-moderate gestational alcohol use and intrauterine growth retardation, low birthweight, and preterm delivery. Ann Epidemiol 7:498-508

9. Amini SA, Dunstan RH, Dunkley PR, Murdoch RN 1996 Oxidative stress and the fetotoxicity of alcohol consumption during pregnancy. Free Radic Biol Med 21:357365

10. Colton CA, Snell-Callanan J, Chernyshev ON 1998 Ethanol induced changes in superoxide anion and nitric oxide in cultured microglia. Alcohol Clin Exp Res 22:710-716

11. Devi BG, Schenker S, Mazloum B, Henderson GI 1996 Ethanol-induced oxidative stress and enzymatic defenses in cultured fetal rat hepatocytes. Alcohol 13:327-332

12. Henderson GI, Devi BG, Perez A, Schenker S 1995 In utero ethanol exposure elicits oxidative stress in the rat fetus. Alcohol Clin Exp Res 19:714-720

13. Ramachandran V, Perez A, Chen J, Senthil D, Schenker S, Henderson GI 2001 In utero ethanol exposure causes mitochondrial dysfunction, which can result in apoptotic cell death in fetal brain: a potential role for 4-hydroxynonenal. Alcohol Clin Exp Res 25:862-871

14. Brooks PJ 1997 DNA damage, DNA repair, and alcohol toxicity-a review. Alcohol Clin Exp Res 21:1073-1082

15. Addolorato G, Gasbarrini A, Marcoccia S, Simoncini M, Baccarini P, Vagni G, Grieco A, Sbriccoli A, Granato A, Stefanini GF, Gasbarrini G 1997 Prenatal exposure to ethanol in rats: effects on liver energy level and antioxidant status in mothers, fetuses, and newborns. Alcohol 14:569-573

16. Reyes E, Ott S, Robinson B 1993 Effects of in utero administration of alcohol on glutathione levels in brain and liver. Alcohol Clin Exp Res 17:877-881

17. Bai C, Brown LA, Jones DP 1994 Glutathione transport by type II cells in perfused rat lung. Am J Physiol 267:L447-L455

18. Jain A, Mehta T, Auld PA, Rodrigues J, Ward RF, Schwartz MK, Martensson J 1995 Glutathione metabolism in newborns: evidence for glutathione deficiency in plasma, bronchoalveolar lavage fluid, and lymphocytes in prematures. Pediatr Pulmonol 20:160-166

19. Smith CV, Hansen TN, Martin NE, McMicken HW, Elliott SJ 1993 Oxidant stress responses in premature infants during exposure to hyperoxia. Pediatr Res 34:360-365

20. Frank L, Sosenko IR 1987 Prenatal development of lung antioxidant enzymes in four species. J Pediatr 110:106-110

21. Frank L, Sosenko IR 1991 Failure of premature rabbits to increase antioxidant enzymes during hyperoxic exposure: increased susceptibility to pulmonary oxygen toxicity compared with term rabbits. Pediatr Res 29:292-296

22. Grigg J, Barber A, Silverman M 1993 Bronchoalveolar lavage fluid glutathione in intubated premature infants. Arch Dis Child 69:49-51

23. Bakker JM, Broug-Holub E, Kroes H, van Rees EP, Kraal G, van Iwaarden JF 1998 Functional immaturity of rat alveolar macrophages during postnatal development. Immunology 94:304-309

24. Bellanti JA, Zeligs BJ 1995 Developmental aspects of pulmonary defenses in children. Pediatr Pulmonol Suppl 11:79-80

25. Kurland G, Cheung AT, Miller ME, Ayin SA, Cho MM, Ford EW 1988 The ontogeny of pulmonary defenses: alveolar macrophage function in neonatal and juvenile rhesus monkeys. Pediatr Res 23:293-297
26. Roos D, Weening RS, Voetman AA, van Schaik ML, Bot AA, Meerhof LJ, Loos JA 1979 Protection of phagocytic leukocytes by endogenous glutathione: studies in a family with glutathione reductase deficiency. Blood 53:851-866

27. Rajkovic IA, Williams R 1985 Inhibition of neutrophil function by hydrogen peroxide. Effect of SH- group-containing compounds. Biochem Pharmacol 34:2083-2090

28. Abdollah S, Brien JF 1995 Effect of chronic maternal ethanol administration on glutamate and $N$-methyl-D-aspartate binding sites in the hippocampus of the near-term fetal guinea pig [published erratum appears in Alcohol 1996;13:107]. Alcohol 12:377-382

29. Kimura KA, Brien JF 1998 Hippocampal nitric oxide synthase in the fetal guinea pig: effects of chronic prenatal ethanol exposure. Brain Res Dev Brain Res 106:39-46

30. Kimura KA, Parr AM, Brien JF 1996 Effect of chronic maternal ethanol administration on nitric oxide synthase activity in the hippocampus of the mature fetal guinea pig. Alcohol Clin Exp Res 20:948-953

31. Brown LA 1994 Glutathione protects signal transduction in type II cells under oxidative stress. Am J Physiol 266:L172-L177

32. Brown LA, Perez JA, Harris FL, Clark RH 1996 Glutathione supplements protect preterm rabbits from oxidative lung injury. Am J Physiol 270:L446-L451

33. Brown LA, Harris FL, Bechara R, Guidot DM 2001 Effect of chronic ethanol ingestion on alveolar type II cell: glutathione and inflammatory mediator-induced apoptosis. Alcohol Clin Exp Res 25:1078-1085

34. Watts CL, Bruce MC 1995 Comparison of secretory component for immunoglobulin A with albumin as reference proteins in tracheal aspirate from preterm infants. J Pediatr 127:113-122

35. Foreman MG, Hoor TT, Brown LA, Moss M 2002 Effects of chronic hepatic dysfunction on pulmonary glutathione homeostasis. Alcohol Clin Exp Res 26:18401845

36. Burnham EL, Brown LA, Halls L, Moss M 2003 Effects of chronic alcohol abuse on alveolar epithelial barrier function and glutathione homeostasis. Alcohol Clin Exp Res 27:1167-1172

37. Moffat FL Jr, Han T, Li ZM, Peck MD, Jy W, Ahn YS, Chu AJ, Bourguignon LY 1996 Supplemental L-arginine $\mathrm{HCl}$ augments bacterial phagocytosis in human polymorphonuclear leukocytes. J Cell Physiol 168:26-33

38. Shen HM, Zhang Z, Zhang QF, Ong CN 2001 Reactive oxygen species and caspase activation mediate silica-induced apoptosis in alveolar macrophages. Am J Physiol Lung Cell Mol Physiol 280:L10-L17

39. Moss M, Guidot DM, Wong-Lambertina M, Ten Hoor T, Perez RL, Brown LA 2000 The effects of chronic alcohol abuse on pulmonary glutathione homeostasis. Am J Respir Crit Care Med 161:414-419

40. Moss M, Steinberg KP, Guidot DM, Duhon GF, Treece P, Wolken R, Hudson LD, Parsons PE 1999 The effect of chronic alcohol abuse on the incidence of ARDS and the severity of the multiple organ dysfunction syndrome in adults with septic shock: an interim and multivariate analysis. Chest 116:97S-98S

41. Holguin F, Moss I, Brown LA, Guidot DM 1998 Chronic ethanol ingestion impairs alveolar type II cell glutathione homeostasis and function and predisposes to endotoxin-mediated acute edematous lung injury in rats. J Clin Invest 101:761-768

42. Guidot D, Moss M, Holguin F, Lois M, Brown L 1999 Ethanol ingestion impairs alveolar epithelial glutathione homeostasis and function, and predisposes to endotoxin-mediated acute lung injury. Chest 116:82S

43. Chen JJ, Schenker S, Henderson GI 1997 4-hydroxynonenal levels are enhanced in fetal liver mitochondria by in utero ethanol exposure. Hepatology 25:142-147

44. Seyoum G, Persaud TV 1994 In vitro effect of S-adenosyl methionine on ethanol embryopathy in the rat. Exp Toxicol Pathol 46:177-181

45. Heaton MB, Mitchell JJ, Paiva M 2000 Amelioration of ethanol-induced neurotoxicity in the neonatal rat central nervous system by antioxidant therapy. Alcohol Clin Exp Res 24:512-518

46. Devi BG, Henderson GI, Frosto TA, Schenker S 1993 Effect of ethanol on rat fetal hepatocytes: studies on cell replication, lipid peroxidation and glutathione. Hepatology 18:648-659

47. Devi BG, Henderson GI, Frosto TA, Schenker S 1994 Effect of acute ethanol exposure on cultured fetal rat hepatocytes: relation to mitochondrial function. Alcohol Clin Exp Res 18:1436-1442

48. Damasceno DC, Volpato GT, de Mattos Paranhos Calderon I, Cunha Rudge MV 2002 Oxidative stress and diabetes in pregnant rats. Anim Reprod Sci 72:235-244

49. Cederberg J, Basu S, Eriksson UJ 2001 Increased rate of lipid peroxidation and protein carbonylation in experimental diabetic pregnancy. Diabetologia 44:766774

50. Gitto E, Reiter RJ, Karbownik M, Tan DX, Gitto P, Barberi S, Barberi I 2002 Causes of oxidative stress in the pre- and perinatal period. Biol Neonate 81:146-157

51. Barden A, Ritchie J, Walters B, Michael C, Rivera J, Mori T, Croft K, Beilin L 2001 Study of plasma factors associated with neutrophil activation and lipid peroxidation in preeclampsia. Hypertension 38:803-808

52. Diedrich F, Renner A, Rath W, Kuhn W, Wieland E 2001 Lipid hydroperoxides and free radical scavenging enzyme activities in preeclampsia and HELLP (hemolysis, elevated liver enzymes, and low platelet count) syndrome: no evidence for circulating primary products of lipid peroxidation. Am J Obstet Gynecol 185:166-172

53. Karowicz-Bilinska A, Suzin J, Sieroszewski P2002 Evaluation of oxidative stress indices during treatment in pregnant women with intrauterine growth retardation. Med Sci Monit 8:CR211-CR216

54. Woods JR Jr 2001 Reactive oxygen species and preterm premature rupture of membranes-a review. Placenta 22:S38-S44 